\title{
HARMONIC HYPERGEOMETRIC FUNCTIONS
}

\author{
R. A. AL-KHAL AND H. A. AL-KHARSANI
}

\begin{abstract}
In this paper we try to uncover some of the inequalities associating hypergeometric functions with planer harmonic mappings. Sharp coefficient relations, distortion theorems and neighborhood are given for these functions. Furthermore, convolution products are considered.
\end{abstract}

\section{Introduction}

Let $U$ denote the open unit disc and $S_{H}$ denote the class of functions which are complex-valued, harmonic, univalent, sense-preserving in $U$ normalized by $f(0)=f_{z}(0)-$ $1=0$. Each $f \in S_{H}$ can be expressed as $f=h+\bar{g}$, where $h$ and $g$ are analytic in $U$. A necessary and sufficient condition for $f$ to be locally univalent and sense-preserving in $U$ is that $\left|h^{\prime}(z)\right|>\left|g^{\prime}(z)\right|$ in $U$. Thus for $f=h+\bar{g} \in S_{H}$ we may write

$$
h(z)=z+\sum_{k=2}^{\infty} A_{k} z^{k}, g(z)=\sum_{k=1}^{\infty} B_{k} z^{k},\left|B_{1}\right|<1 .
$$

Clunie and Sheil-Small [4] studied the class $S_{H}$ with some geometric subclasses of $S_{H}$.

Let $H P(\beta)$ denote the subclass of $S_{H}$ satisfying $\operatorname{Re}\left\{h^{\prime}(z)+g^{\prime}(z)\right\}>\beta, \quad 0 \leq \beta<1$ which was studied by Yalçin et al. [8]; they also denoted by $\operatorname{HP}^{*}(\beta)$ the subclass of $H P(\beta)$ such that the functions $h$ and $g$ in $f=h+\bar{g}$ are of the form

$$
h(z)=z-\sum_{k=2}^{\infty}\left|A_{k}\right| z^{k} \text { and } g(z)=-\sum_{k=1}^{\infty}\left|B_{k}\right| z^{k} .
$$

If $f_{j}=h_{j}+\bar{g}_{j}, \quad j=1,2, \ldots$ are in the class $S_{H}$, then we define the convolution $f_{1} * f_{2}$ of $f_{1}$ and $f_{2}$ in the natural way $h_{1} * h_{2}+\overline{g_{1} * g_{2}}$. If $\phi_{1}$ and $\phi_{2}$ are analytic and $f=h+\bar{g}$ is in $S_{H}$, Ahuja and Silverman [6] defined

$$
f \tilde{*}\left(\phi_{1}+\bar{\phi}_{2}\right)=h * \phi_{1}+\overline{g * \phi_{2}} .
$$

Received August 8, 2005; revised December 21, 2005

2000 Mathematics Subject Classification. Primary 30C55; Secondary 31A05, 33C90.

Key words and phrases. Planer harmonic mappings, hypergeometric functions, convolution multipliers, harmonic functions, distortion bounds. 
Let $a, b, c$ be complex numbers with $c \neq 0,-1,-2, \ldots$ Then the Gauss hypergeometric function written as ${ }_{2} F_{1}(a, b ; c ; z)$ or simply as $F(a, b ; c ; z)$ is defined by

$$
F(a, b ; c ; z)=\sum_{k=0}^{\infty} \frac{(a)_{k}(b)_{k}}{(c)_{k}(1)_{k}} z^{k}
$$

where $(\lambda)_{k}$ is the Pochhammer symbol defined by

$$
(\lambda)_{k}=\frac{\Gamma(\lambda+k)}{\Gamma(\lambda)}=\lambda(\lambda+1) \cdots(\lambda+k-1) \text { for } k=1,2,3, \ldots, \quad \text { and }(\lambda)_{0}=1 .
$$

Since the hypergeometric series in (1.4) converges absolutely in $U$, it follows that $F(a, b ; c ; z)$ defines a function which is analytic in $U$, provided that $c$ is neither zero nor a negative integer. In terms of Gamma functions, we are led to the well-known Gauss's summation theorem: If $\operatorname{Re}(c-a-b)>0$, then

$$
F(a, b ; c ; 1)=\frac{\Gamma(c) \Gamma(c-a-b)}{\Gamma(c-a) \Gamma(c-b)}, \quad c \neq 0,-1,-2, \ldots
$$

For further information about hypergeometric functions, one may refer to [1], [2], and [3].

Throughout this paper, let $G(z):=\phi_{1}(z)+\overline{\phi_{2}(z)}$ be a function where $\phi_{1}(z) \equiv$ $\phi_{1}\left(a_{1}, b_{1} ; c_{1} ; z\right)$ and $\phi_{2}(z) \equiv \phi_{2}\left(a_{2}, b_{2} ; c_{2} ; z\right)$ are the hypergeometric functions defined by

$$
\begin{aligned}
& \phi_{1}(z)=z F\left(a_{1}, b_{1} ; c_{1} ; z\right)=z+\sum_{k=2}^{\infty} \frac{\left(a_{1}\right)_{k-1}\left(b_{1}\right)_{k-1}}{\left(c_{1}\right)_{k-1}(1)_{k-1}} z^{k}, \\
& \phi_{2}(z) z F\left(a_{2}, b_{2} ; c_{2} ; z\right)-1=\sum_{k=1}^{\infty} \frac{\left(a_{2}\right)_{k}\left(b_{2}\right)_{k}}{\left(c_{2}\right)_{k}(1)_{k}} z^{k}, \quad a_{2} b_{2}<c_{2}, a_{j}, b_{j}, c_{j} \text { are } \\
& \quad \text { positive for } j=1,2 .
\end{aligned}
$$

The purpose of this paper is to uncover some of the connections between the theory of harmonic univalent functions and hypergeometric functions. We will investigate the convolution multipliers $f \tilde{*}\left(\phi_{1}+\bar{\phi}_{2}\right)$ and the neighborhood of $G=\phi_{1}+\bar{\phi}_{2}$, where $\phi_{1}, \phi_{2}$ are as defined by (1.7), (1.8) and $f \in H P(\beta)$. Also, convolution products are considered.

\section{Preliminary Results}

In order to derive new results, we need the following lemmas due to Yalçin et al. [8].

Lemma 2.1. For $f=h+\bar{g}$ with $h$ and $g$ of the form (1.1), if

$$
\sum_{k=1}^{\infty} k\left(\left|A_{k}\right|+\left|B_{k}\right|\right) \leq 2-\beta
$$


where $A_{1}=1$ and $0 \leq \beta<1$, then $f \in H P(\beta)$.

Lemma 2.2. For $f=h+\bar{g}$ with $h$ and $g$ of the form (1.2). Then $f \in H P^{*}(\beta)$ if and only if

$$
\left.\sum_{k=1}^{\infty} k\left(\left|A_{k}\right|\right)+\left|B_{k}\right|\right) \leq 2-\beta .
$$

where $A_{1}=1$ and $0 \leq \beta<1$.

Lemma 2.3. If $f \in H P^{*}(\beta)$, then

$$
|f(z)| \leq\left(1+\left|B_{1}\right|\right) r+\frac{1}{2}\left(1-\left|B_{1}\right|-\beta\right) r^{2}, \quad|z|=r<1,
$$

and

$$
|f(z)| \geq\left(1-\left|B_{1}\right|\right) r-\frac{1}{2}\left(1-\left|B_{1}\right|-\beta\right) r^{2}, \quad|z|=r<1 .
$$

Hence

$$
\left\{\omega:|\omega|<\frac{1}{2}\left(1-\left|B_{1}\right|+\beta\right)\right\} \subset f(U) .
$$

\section{Main Results}

Theorem 3.1. If $c_{j}>a_{j}+b_{j}+1$ for $j=1,2$, then a sufficient condition for $G=\phi_{1}+\bar{\phi}_{2}$ to be harmonic univalent in $U$ and $G \in H P(\beta), \quad 0 \leq \beta<1$ is that

$$
\left(1+\frac{a_{1} b_{1}}{c_{1}-a_{1}-b_{1}-1}\right) F\left(a_{1}, b_{1} ; c_{1} ; 1\right)+\frac{a_{2} b_{2}}{c_{2}-a_{2}-b_{2}-1} F\left(a_{2}, b_{2} ; c_{2} ; 1\right) \leq 2-\beta .
$$

Proof. In order to prove that $G$ is locally univalent and sense-preserving in $U$, we only need to show that $\left|\phi_{1}^{\prime}(z)\right|>\left|\phi_{2}^{\prime}(z)\right|, \quad z \in U$. In view of (1.4), (1.5), (1.6) and (1.7), we have

$$
\begin{aligned}
\left|\phi_{1}^{\prime}(z)\right| & =\left|1+\sum_{k=2}^{\infty} k \frac{\left(a_{1}\right)_{k-1}\left(b_{1}\right)_{k-1}}{\left(c_{1}\right)_{k-1}(1)_{k-1}} z^{k-1}\right| \\
& >1-\sum_{k=2}^{\infty}(k-1) \frac{\left(a_{1}\right)_{k-1}\left(b_{1}\right)_{k-1}}{\left(c_{1}\right)_{k-1}(1)_{k-1}}-\sum_{k=2}^{\infty} \frac{\left(a_{1}\right)_{k-1}\left(b_{1}\right)_{k-1}}{\left(c_{1}\right)_{k-1}(1)_{k-1}} \\
& =1-\frac{a_{1} b_{1}}{c_{1}} \sum_{k=2}^{\infty} \frac{\left(a_{1}+1\right)_{k-2}\left(b_{1}+1\right)_{k-2}}{\left(c_{1}+1\right)_{k-2}(1)_{k-2}}-\sum_{k=1}^{\infty} \frac{\left(a_{1}\right)_{k}\left(b_{1}\right)_{k}}{\left(c_{1}\right)_{k}(1)_{k}} \\
& =1-\frac{a_{1} b_{1}}{c_{1}} \sum_{k=1}^{\infty} \frac{\left(a_{1}+1\right)_{k-1}\left(b_{1}+1\right)_{k-1}}{\left(c_{1}+1\right)_{k-1}(1)_{k-1}}-\sum_{k=1}^{\infty} \frac{\left(a_{1}\right)_{k}\left(b_{1}\right)_{k}}{\left(c_{1}\right)_{k}(1)_{k}} \\
& =2-\frac{a_{1} b_{1}}{c_{1}} \frac{\Gamma\left(c_{1}+1\right) \Gamma\left(c_{1}-a_{1}-b_{1}-1\right)}{\Gamma\left(c_{1}-a_{1}\right) \Gamma\left(c_{1}-b_{1}\right)}-F\left(a_{1}, b_{1} ; c_{1} ; 1\right) \\
& =2-\left(\frac{a_{1} b_{1}}{c_{1}-a_{1}-b_{1}-1}+1\right) F\left(a_{1}, b_{1} ; c_{1} ; 1\right) .
\end{aligned}
$$


Again, using (3.1), (1.4), (1.6) and (1.8) in turn, to the above mentioned inequality, we have

$$
\begin{aligned}
\left|\phi_{1}^{\prime}(z)\right| & \geq \beta+\frac{a_{2} b_{2}}{c_{2}-a_{2}-b_{2}-1} F\left(a_{2}, b_{2} ; c_{2} ; 1\right) \\
& \geq \frac{a_{2} b_{2}}{c_{2}-a_{2}-b_{2}-1} F\left(a_{2}, b_{2} ; c_{2} ; 1\right) \\
& =\frac{a_{2} b_{2}}{c_{2}} \frac{\Gamma\left(c_{2}+1\right) \Gamma\left(c_{2}-a_{2}-b_{2}-1\right)}{\Gamma\left(c_{2}-a_{2}\right) \Gamma\left(c_{2}-b_{2}\right)} \\
& =\sum_{k=0}^{\infty} \frac{\left(a_{2}\right)_{k+1}\left(b_{2}\right)_{k+1}}{\left(c_{2}\right)_{k+1}(1)_{k}} \\
& >\sum_{k=1}^{\infty} k \frac{\left(a_{2}\right)_{k}\left(b_{2}\right)_{k}}{\left(c_{2}\right)_{k}(1)_{k}}|z|^{k-1} \\
& \geq\left|\sum_{k=1}^{\infty} k \frac{\left(a_{2}\right)_{k}\left(b_{2}\right)_{k}}{\left(c_{2}\right)_{k}(1)_{k}} z^{k-1}\right|=\left|\phi_{2}^{\prime}(z)\right| .
\end{aligned}
$$

To show that $G$ is univalent in $U$, we assume that $z_{1}, z_{2} \in U$ so that $z_{1} \neq z_{2}$. Since $U$ is simply connected and convex, we have $z(t)=(1-t) z_{1}+t z_{2} \in U$, where $0 \leq t \leq 1$. Then we can write

$$
G\left(z_{2}\right)-G\left(z_{1}\right)=\int_{0}^{1}\left[\left(z_{2}-z_{1}\right) \phi_{1}^{\prime}(z(t))+\overline{\left(z_{2}-z_{1}\right) \phi_{2}^{\prime}(z(t))}\right] d t
$$

so that

$$
\begin{aligned}
\operatorname{Re} \frac{G\left(z_{2}\right)-G\left(z_{1}\right)}{z_{2}-z_{1}} & =\int_{0}^{1} \operatorname{Re}\left[\phi_{1}^{\prime}(z(t))+\frac{\overline{\left(z_{2}-z_{1}\right)}}{z_{2}-z_{1}} \phi_{2}^{\prime}(z(t))\right] d t \\
& >\int_{0}^{1}\left[\operatorname{Re} \phi_{1}^{\prime}(z(t))-\left|\phi_{2}^{\prime}(z(t))\right|\right] d t
\end{aligned}
$$

On the other hand,

$$
\begin{aligned}
& \operatorname{Re} \phi_{1}^{\prime}(z)-\left|\phi_{2}^{\prime}(z)\right| \\
& \geq 1-\sum_{k=2}^{\infty} k \frac{\left(a_{1}\right)_{k-1}\left(b_{1}\right)_{k-1}}{\left(c_{1}\right)_{k-1}(1)_{k-1}}|z|^{k-1}-\sum_{k=1}^{\infty} k \frac{\left(a_{2}\right)_{k}\left(b_{2}\right)_{k}}{\left(c_{2}\right)_{k}(1)_{k}}|z|^{k-1} \\
& >1-\sum_{k=2}^{\infty}(k-1+1) \frac{\left(a_{1}\right)_{k-1}\left(b_{1}\right)_{k-1}}{\left(c_{1}\right)_{k-1}(1)_{k-1}}-\sum_{k=1}^{\infty} k \frac{\left(a_{2}\right)_{k}\left(b_{2}\right)_{k}}{\left(c_{2}\right)_{k}(1)_{k}} \\
& =2-\sum_{k=2}^{\infty} \frac{\left(a_{1}\right)_{k-1}\left(b_{1}\right)_{k-1}}{\left(c_{1}\right)_{k-1}(1)_{k-2}}-\sum_{k=0}^{\infty} \frac{\left(a_{1}\right)_{k}\left(b_{1}\right)_{k}}{\left(c_{1}\right)_{k}(1)_{k}}-\frac{a_{2} b_{2}}{c_{2}} \sum_{k=1}^{\infty} \frac{\left(a_{2}+1\right)_{k-1}\left(b_{2}+1\right)_{k-1}}{\left(c_{2}+1\right)_{k-1}(1)_{k-1}}
\end{aligned}
$$




$$
\begin{aligned}
& =2-\left(1+\frac{a_{1} b_{1}}{c_{1}-a_{1}-b_{1}-1}\right) F\left(a_{1}, b_{1} ; c_{1} ; 1\right)-\frac{a_{2} b_{2}}{c_{2}-a_{2}-b_{2}-1} F\left(a_{2}, b_{2} ; c_{2} ; 1\right) \\
& \geq \beta \\
& \geq 0, \quad \text { by }(3.1) .
\end{aligned}
$$

Thus (3.2) and the above inequality lead to $G\left(z_{1}\right) \neq G\left(z_{2}\right)$ and hence $G$ is univalent in $U$. In order to prove that $G \in H P(\beta)$, using Lemma 2.1, we only need to prove that

$$
\sum_{k=1}^{\infty} k\left(\frac{\left(a_{1}\right)_{k-1}\left(b_{1}\right)_{k-1}}{\left(c_{1}\right)_{k-1}(1)_{k-1}}+\frac{\left(a_{2}\right)_{k}\left(b_{2}\right)_{k}}{\left(c_{2}\right)_{k}(1)_{k}}\right) \leq 2-\beta
$$

or

$$
\sum_{k=2}^{\infty} k \frac{\left(a_{1}\right)_{k-1}\left(b_{1}\right)_{k-1}}{\left(c_{1}\right)_{k-1}(1)_{k-1}}+\sum_{k=1}^{\infty} k \frac{\left(a_{2}\right)_{k}\left(b_{2}\right)_{k}}{\left(c_{2}\right)_{k}(1)_{k}} \leq 1-\beta .
$$

Writing $k=k-1+1$, the left hand side of (3.3) reduces to

$$
\begin{aligned}
\frac{a_{1} b_{1}}{c_{1}} \sum_{k=0}^{\infty} & \frac{\left(a_{1}+1\right)_{k}\left(b_{1}+1\right)_{k}}{\left(c_{1}+1\right)_{k}(1)_{k}}+\left[\sum_{k=0}^{\infty} \frac{\left(a_{1}\right)_{k}\left(b_{1}\right)_{k}}{\left(c_{1}\right)_{k}(1)_{k}}-1\right] \\
& +\frac{a_{2} b_{2}}{c_{2}} \sum_{k=0}^{\infty} \frac{\left(a_{2}+1\right)_{k}\left(b_{2}+1\right)_{k}}{\left(c_{2}+1\right)_{k}(1)_{k}} \\
& =\left(\frac{a_{1} b_{1}}{c_{1}-a_{1}-b_{1}-1}+1\right) F\left(a_{1}, b_{1} ; c_{1} ; 1\right)+\frac{a_{2} b_{2}}{c_{2}-a_{2}-b_{2}-1} F\left(a_{2}, b_{2} ; c_{2} ; 1\right)-1 .
\end{aligned}
$$

The last expression is bounded above by $1-\beta$ so that (3.1) is satisfied. This completes the proof

Theorem 3.2. Let $c_{j}>a_{j}+b_{j}+1$, for $j=1,2$ and $a_{2} b_{2}<c_{2}$. If $G=\phi_{1}(z)+\overline{\phi_{2}(z)}$ with

$$
\begin{aligned}
& \phi_{1}(z)=z-\sum_{k=2}^{\infty} \frac{\left(a_{1}\right)_{k-1}\left(b_{1}\right)_{k-1}}{\left(c_{1}\right)_{k-1}(1)_{k-1}} z^{k} \\
& \phi_{2}(z)=1-z F\left(a_{2}, b_{2} ; c_{2} ; z\right)=-\sum_{k=1}^{\infty} \frac{\left(a_{2}\right)_{k}\left(b_{2}\right)_{k}}{\left(c_{2}\right)_{k}(1)_{k}} z^{k},
\end{aligned}
$$

then $G \in H P^{*}(\beta), \quad 0 \leq \beta<1$ if and only if (3.1) holds.

Proof. We observe that $H P^{*}(\beta) \subset H P(\beta)$. In view of Theorem 3.1, we need only to show the necessary condition for $G$ to be in $H P(\beta)$. If $G \in H P^{*}(\beta)$, then $G$ satisfies the inequality in Lemma 2.2, and the result follows.

Theorem 3.3. Let $a_{2} b_{2}<c_{2}$ and $G$ of the form (3.4). If $G \in H P^{*}(\beta)$, then

$$
|G(z)| \leq\left(1+\frac{a_{2} b_{2}}{c_{2}}\right) r+\frac{1}{2}\left(1-\frac{a_{2} b_{2}}{c_{2}}-\beta\right) r^{2}, \quad|z|=r<1
$$


and

$$
|G(z)| \geq\left(1-\frac{a_{2} b_{2}}{c_{2}}\right) r-\frac{1}{2}\left(1-\frac{a_{2} b_{2}}{c_{2}}-\beta\right) r^{2}, \quad|z|=r<1 .
$$

Hence

$$
\left\{\omega:|\omega|<\frac{1}{2}\left(1-\frac{a_{2} b_{2}}{c_{2}}+\beta\right)\right\} \subset G(U) .
$$

Proof. The result follows from Lemma 2.3.

Theorem 3.4. Let $c_{j}>a_{j}+b_{j}+1$ for $j=1,2$ and $a_{2} b_{2}<c_{2}$. A necessary and sufficient condition such that $f \tilde{*}\left(\phi_{1}+\overline{\phi_{2}}\right) \in H P^{*}(\beta)$ for $f \in H P^{*}(\beta)$ is that

$$
F\left(a_{1}, b_{1} ; c_{1} ; 1\right)+F\left(a_{2}, b_{2} ; c_{2} ; 1\right) \leq 3,
$$

where $\phi_{1}, \phi_{2}$ are as defined, respectively, by (1.7) and (1.8)

Proof. Let $f=h+\bar{g} \in H P^{*}(\beta)$, where $h$ and $g$ are given by (1.2). Then

$$
\begin{aligned}
\left(f \tilde{*}\left(\phi_{1}+\bar{\phi}_{2}\right)\right)(z)= & h(z) * \phi_{1}(z)+\overline{g(z) * \phi_{2}(z)} \\
= & z-\sum_{k=2}^{\infty} \frac{\left(a_{1}\right)_{k-1}\left(b_{1}\right)_{k-1}}{\left(c_{1}\right)_{k-1}(1)_{k-1}}\left|A_{k}\right| z^{k} \\
& \frac{-\sum_{k=1}^{\infty} \frac{\left(a_{2}\right)_{k}\left(b_{2}\right)_{k}}{\left(c_{2}\right)_{k}(1)_{k}}\left|B_{k}\right| z^{k}}{}
\end{aligned}
$$

In view of Lemma 2.2, we need to prove that $f *\left(\phi_{1}+\bar{\phi}_{2}\right) \in H P^{*}(\beta)$ if and only if

$$
\sum_{k=1}^{\infty} k\left[\frac{\left(a_{1}\right)_{k-1}\left(b_{1}\right)_{k-1}}{\left(c_{1}\right)_{k-1}(1)_{k-1}}\left|A_{k}\right|+\frac{\left(a_{2}\right)_{k}\left(b_{2}\right)_{k}}{\left(c_{2}\right)_{k}(1)_{k}}\left|B_{k}\right|\right] \leq 2-\beta
$$

or

$$
\sum_{k=2}^{\infty} k \frac{\left(a_{1}\right)_{k-1}\left(b_{1}\right)_{k-1}}{\left(c_{1}\right)_{k-1}(1)_{k-1}}\left|A_{k}\right|+\sum_{k=1}^{\infty} k \frac{\left(a_{2}\right)_{k}\left(b_{2}\right)_{k}}{\left(c_{2}\right)_{k}(1)_{k}}\left|B_{k}\right| \leq 1-\beta .
$$

As an application of Lemma 2.2, we have

$$
\left|A_{k}\right| \leq \frac{1-\beta}{k}, \quad\left|B_{k}\right| \leq \frac{1-\beta}{k}
$$

Therefore, the left hand side of (3.6) is bounded above by

$$
\begin{aligned}
\sum_{k=2}^{\infty}(1-\beta) \frac{\left(a_{1}\right)_{k-1}\left(b_{1}\right)_{k-1}}{\left(c_{1}\right)_{k-1}(1)_{k-1}} & +\sum_{k=1}^{\infty}(1-\beta) \frac{\left(a_{2}\right)_{k}\left(b_{2}\right)_{k}}{\left(c_{2}\right)_{k}(1)_{k}} \\
& =(1-\beta)\left[F\left(a_{1}, b_{1} ; c_{1} ; 1\right)+F\left(a_{2}, b_{2} ; c_{2} ; 1\right)-2\right]
\end{aligned}
$$


The last expression is bounded above by $(1-\beta)$ if and only if $(3.5)$ is satisfied. This proves (3.6) and the result follows.

Theorem 3.5. If $c_{j}>a_{j}+b_{j}$ for $j=1,2$, then a sufficient condition for a function

$$
G_{1}(z)=\int_{0}^{z} F\left(a_{1}, b_{1} ; c_{1} ; t\right) d t+\overline{\int_{0}^{z}\left[F\left(a_{2}, b_{2} ; c_{2} ; t\right)-1\right]} d t
$$

to be in $H P(\beta)$ is that

$$
F\left(a_{1}, b_{1} ; c_{1} ; 1\right)+F\left(a_{2}, b_{2} ; c_{2} ; 1\right) \leq 3-\beta, \quad 0 \leq \beta<1 .
$$

Proof. In view of Lemma 2.1, the function

$$
G_{1}(z)=z+\sum_{k=2}^{\infty} \frac{\left(a_{1}\right)_{k-1}\left(b_{1}\right)_{k-1}}{\left(c_{1}\right)_{k-1}(1)_{k}} z^{k}+\overline{\sum_{k=2}^{\infty} \frac{\left(a_{2}\right)_{k-1}\left(b_{2}\right)_{k-1}}{\left(c_{2}\right)_{k-1}(1)_{k}} z^{k}}
$$

is in $H P(\beta)$ if

$$
\sum_{k=2}^{\infty} k \frac{\left(a_{1}\right)_{k-1}\left(b_{1}\right)_{k-1}}{\left(c_{1}\right)_{k-1}(1)_{k}}+\sum_{k=2}^{\infty} k \frac{\left(a_{2}\right)_{k-1}\left(b_{2}\right)_{k-1}}{\left(c_{2}\right)_{k-1}(1)_{k}} \leq 1-\beta .
$$

That is, if

$$
\sum_{k=1}^{\infty} \frac{\left(a_{1}\right)_{k}\left(b_{1}\right)_{k}}{\left(c_{1}\right)_{k}(1)_{k}}+\sum_{k=1}^{\infty} \frac{\left(a_{2}\right)_{k}\left(b_{2}\right)_{k}}{\left(c_{2}\right)_{k}(1)_{k}} \leq 1-\beta .
$$

Equivalently, $G_{1} \in H P(\beta)$ if

$$
F\left(a_{1}, b_{1} ; c_{1} ; 1\right)+F\left(a_{2}, b_{2} ; c_{2} ; 1\right) \leq 3-\beta .
$$

In the next theorem we give a necessary and sufficient convolution condition for $G=\phi_{1}+\bar{\phi}_{2}$ to be in $H P(\beta)$.

Theorem 3.6. Let $a_{2} b_{2}<c_{2}$. If $G=\phi_{1}(z)+\bar{\phi}_{2}(z)$ with $\phi_{1}, \phi_{2}$ are as defined, respectively, by (1.7) and (1.8). Then $G \in H P(\beta)$ if and only if $\left(\left(\phi_{1}+\phi_{2}\right) * \frac{(\xi+1)}{(1-z)^{2}}\right)+\frac{a_{2} b_{2}+c_{2}}{c_{2}}(1-2 \beta-\xi) \neq 0, \quad 0 \leq \beta<1,|\xi|=1, \quad 0<|z|<1$.

Proof. Let $G \in H P(\beta)$, then

$$
\operatorname{Re}\left\{\phi_{1}^{\prime}+\phi_{2}^{\prime}\right\}>\beta
$$


Since $\frac{c_{2}}{a_{2} b_{2}+c_{2}}\left(\phi_{1}^{\prime}(z)+\phi_{2}^{\prime}(z)\right)=1$ at $z=0$, therefore we can write $G \in H P(\beta)$ if and only if

$$
\frac{1}{1-\beta}\left[\frac{c_{2}}{a_{2} b_{2}+c_{2}}\left(\phi_{1}^{\prime}(z)+\phi_{2}^{\prime}(z)\right)-\beta\right] \neq \frac{\xi-1}{\xi+1}, \quad|\xi|=1, \quad \xi \neq-1,0<|z|<1 .
$$

By a simple algebraic manipulation, we get

$$
\begin{aligned}
0 & \neq(\xi+1) \frac{c_{2}}{a_{2} b_{2}+c_{2}}\left(\phi_{1}^{\prime}(z)+\phi_{2}^{\prime}(z)\right)-\beta(\xi+1)-(1-\beta)(\xi-1) \\
& =\frac{c_{2}}{a_{2} b_{2}+c_{2}}(\xi+1)\left(\phi_{1}^{\prime}(z)+\phi_{2}^{\prime}(z)\right)+(1-2 \beta-\xi) \\
& =\frac{c_{2}}{a_{2} b_{2}+c_{2}}\left(\phi_{1}(z)+\phi_{2}(z)\right) * \frac{(\xi+1)}{(1-z)^{2}}+(1-2 \beta-\xi)
\end{aligned}
$$

which is the condition required by Theorem 3.6.

Theorem 3.7. Let $f \in H P^{*}(\beta), \quad 0 \leq \beta<1$. Then the function $H=f \widetilde{*}\left(\phi_{1}+\bar{\phi}_{2}\right)$ is starlike of order $\gamma(0 \leq \gamma<1)$ in $|z|<R$, where

$$
R=\inf _{k}\left[\frac{2 k(1-\gamma)\left(c_{1}\right)_{k-1}\left(c_{2}\right)_{k} k !}{(1-\beta)\left[k(k-\gamma)\left(a_{1}\right)_{k-1}\left(b_{1}\right)_{k-1}\left(c_{2}\right)_{k}+(k+\gamma-2)\left(a_{2}\right)_{k}\left(b_{2}\right)_{k}\left(c_{1}\right)_{k-1}\right]}\right]^{\frac{1}{k-1}}
$$

$\phi_{1}, \phi_{2}$ are as defined by (1.7) and (1.8).

Proof. It is sufficient to show that

$$
\left|\frac{z H^{\prime}}{H}-1\right|<1-\gamma \text { in }|z|<R
$$

For the left hand side of (3.7), we have

$$
\left|\frac{z H^{\prime}}{H}-1\right| \leq \frac{\sum_{k=2}^{\infty} \frac{(k-1)\left(a_{1}\right)_{k-1}\left(b_{1}\right)_{k-1}}{\left(c_{1}\right)_{k-1}(1)_{k-1}}\left|A_{k}\right||z|^{k-1}+\sum_{k=1}^{\infty} \frac{(k+1)\left(a_{2}\right)_{k}\left(b_{2}\right)_{k}}{\left(c_{2}\right)_{k}(1)_{k}}\left|B_{k}\right||z|^{k-1}}{1-\sum_{k=2}^{\infty} \frac{\left(a_{1}\right)_{k-1}\left(b_{1}\right)_{k-1}}{\left(c_{1}\right)_{k-1}(1)_{k-1}}\left|A_{k}\right||z|^{k-1}-\sum_{k=1}^{\infty} \frac{\left(a_{2}\right)_{k}\left(b_{2}\right)_{k}}{\left(c_{2}\right)_{k}(1)_{k}}\left|B_{k}\right||z|^{k-1}} .
$$

The last expression is less than $1-\gamma$ if

$$
\sum_{k=2}^{\infty} \frac{(k-\gamma)}{(1-\gamma)} \frac{\left(a_{1}\right)_{k-1}\left(b_{1}\right)_{k-1}}{\left(c_{1}\right)_{k-1}(1)_{k-1}}\left|A_{k}\right||z|^{k-1}+\sum_{k=1}^{\infty} \frac{(k+2-\gamma)}{(1-\gamma)} \frac{\left(a_{2}\right)_{k}\left(b_{2}\right)_{k}}{\left(c_{2}\right)_{k}(1)_{k}}\left|B_{k}\right||z|^{k-1}<1 .
$$

Using the fact that $f \in H P^{*}(\beta)$ if and only if

$$
\sum_{k=2}^{\infty} k\left|A_{k}\right|+\sum_{k=1}^{\infty} k\left|B_{k}\right| \leq 1-\beta,
$$

we can say (3.8) is true if

$$
\left\{\frac{k-\gamma}{1-\gamma} \frac{\left(a_{1}\right)_{k-1}\left(b_{1}\right)_{k-1}}{\left(c_{1}\right)_{k-1}(1)_{k-1}}+\frac{k+2-\gamma}{1-\gamma} \frac{\left(a_{2}\right)_{k}\left(b_{2}\right)_{k}}{\left(c_{2}\right)_{k}(1)_{k}}\right\}|z|^{k-1}<\frac{2 k}{1-\beta} .
$$


Or equivalently,

$$
\begin{aligned}
|z|^{k-1} & <\frac{2 k(1-\gamma)}{1-\beta}\left\{\frac{\left(c_{1}\right)_{k-1}(1)_{k-1}\left(c_{2}\right)_{k}(1)_{k}}{(k-\gamma)\left(a_{1}\right)_{k-1}\left(b_{1}\right)_{k-1}\left(c_{2}\right)_{k}(1)_{k}+(k+2-\gamma)\left(a_{2}\right)_{k}\left(b_{2}\right)_{k}\left(c_{1}\right)_{k-1}(1)_{k-1}}\right\} \\
& =\frac{2 k(1-\gamma)}{1-\beta}\left\{\frac{k !\left(c_{1}\right)_{k-1}\left(c_{2}\right)_{k}}{k(k-\gamma)\left(a_{1}\right)_{k-1}\left(b_{1}\right)_{k-1}\left(c_{2}\right)_{k}+(k+2-\gamma)\left(a_{2}\right)_{k}\left(b_{2}\right)_{k}\left(c_{1}\right)_{k-1}}\right\} .
\end{aligned}
$$

If $h, g, H, G$ are of the form (1.1) and as we know if $f=h+\bar{g}$ and $F=H+\bar{G}$, then the convolution of $f$ and $F$ is defined to be the function

$$
f * F(z)=z+\sum_{k=2}^{\infty} a_{k} A_{k} z^{k}+\overline{\sum_{k=1}^{\infty} b_{k} B_{k} z^{k}}
$$

while the integral convolution is defined by

$$
f \diamond F(z)=z+\sum_{k=2}^{\infty} \frac{a_{k} A_{k}}{k} z^{k}+\overline{\sum_{k=1}^{\infty} \frac{b_{k} B_{k}}{k} z^{k}} .
$$

The $\delta$-neighborhood of $f$ is the set

$$
N_{\delta}(f)=\left\{F=z+\sum_{k=2}^{\infty} A_{k} z^{k}+\overline{\sum_{k=1}^{\infty} B_{k} z^{k}}: \sum_{k=2}^{\infty} k\left(\left|a_{k}-A_{k}\right|+\left|b_{k}-B_{k}\right|\right)+\left|b_{1}-B_{1}\right| \leq \delta\right\}
$$

(see [5], [7]). In this case, let us define the generalized $\delta$-neighborhood of $G=\phi_{1}+\bar{\phi}_{2}$, where $\phi_{1}, \phi_{2}$ of the form (1.7) and (1.8) to be the set

$$
\begin{aligned}
N_{\delta}(G)= & \left\{F=z+\sum_{k=2}^{\infty} \frac{\left(A_{1}\right)_{k-1}\left(B_{1}\right)_{k-1}}{\left(C_{1}\right)_{k-1}(1)_{k-1}} z^{k}+\sum_{k=1}^{\infty} \frac{\left(A_{2}\right)_{k}\left(B_{2}\right)_{k}}{\left(C_{2}\right)_{k}(1)_{k}} z^{k}:\right. \\
& \sum_{k=2}^{\infty} k\left\{\left(\frac{\left(a_{1}\right)_{k-1}\left(b_{1}\right)_{k-1}}{\left(c_{1}\right)_{k-1}(1)_{k-1}}-\frac{\left(A_{1}\right)_{k-1}\left(B_{1}\right)_{k-1}}{\left(C_{1}\right)_{k-1}(1)_{k-1}}\right)+\left(\frac{\left(a_{2}\right)_{k}\left(b_{2}\right)_{k}}{\left(c_{2}\right)_{k}(1)_{k}}-\frac{\left.\left(A_{2}\right)_{k}\left(B_{2}\right)_{k}\right)}{\left(C_{2}\right)_{k}(1)_{k}}\right)\right\} \\
& +\left(\frac{a_{2} b_{2}}{c_{2}}-\frac{A_{2} B_{2}}{C_{2}}\right) \leq(1-\beta) \delta .
\end{aligned}
$$

Let $P_{H}^{0}$ denote the class of functions $F$ complex and harmonic in $U, F=h+\bar{g}$ such that $\operatorname{Re} F(z)>0, \quad z \in U$ and

$$
h(z)=1+\sum_{k=1}^{\infty} A_{k} z^{k}, \quad g(z)=\sum_{k=2}^{\infty} B_{k} z^{k}
$$

It is known [9, Theorem 3] that the sharp inequalities $\left|A_{k}\right| \leq k+1, \quad\left|B_{k}\right| \leq k-1$ are true. 
Theorem 3.8. (i) If $G=\phi_{1}(z)+\overline{\phi_{2}(z)} \in H P(\beta)$ where $\phi_{1}, \phi_{2}$ are as defied by (1.7) and (1.8), $(0 \leq \beta<1)$, then for $\frac{3}{2} \leq\left|A_{1}\right| \leq 2, \frac{1}{A_{1}} G \diamond F \in H P(\beta)$.

(ii) If $G$ satisfies the condition

$$
\sum_{k=2}^{\infty} k^{2}\left(\frac{\left(a_{1}\right)_{k-1}\left(b_{1}\right)_{k-1}}{\left(c_{1}\right)_{k-1}(1)_{k-1}}+\frac{\left(a_{2}\right)_{k}\left(b_{2}\right)_{k}}{\left(c_{2}\right)_{k}(1)_{k}}\right) \leq 1-\beta
$$

then

$$
\frac{1}{A_{1}} G * F \in H P(\beta)
$$

(iii) If $G$ satisfies (3.9) and $\delta \leq \frac{1}{2}-\frac{1}{1-\beta} \frac{a_{2} b_{2}}{c_{2}}$, then $N_{\delta}(G) \subset H P(\beta)$, where $0 \leq$ $\beta<1, \quad a_{2} b_{2}<c_{2}$.

Proof. We justify the case (ii). Since

$$
\begin{aligned}
\sum_{k=2}^{\infty} k & \left(\frac{\left(a_{1}\right)_{k-1}\left(b_{1}\right)_{k-1}}{\left(c_{1}\right)_{k-1}(1)_{k-1}}\left|\frac{A_{k}}{A_{1}}\right|+\frac{\left(a_{2}\right)_{k}\left(b_{2}\right)_{k}}{\left(c_{2}\right)_{k}(1)_{k}}\left|\frac{B_{k}}{A_{1}}\right|\right) \\
& \leq \sum_{k=2}^{\infty} k^{2}\left(\frac{\left(a_{1}\right)_{k-1}\left(b_{1}\right)_{k-1}}{\left(c_{1}\right)_{k-1}(1)_{k-1}} \frac{k+1}{\left|A_{1}\right| k}+\frac{\left(a_{2}\right)_{k}\left(b_{2}\right)_{k}}{\left(c_{2}\right)_{k}(1)_{k}} \frac{k-1}{\left|A_{1}\right| k}\right) \\
& \leq \sum_{k=2}^{\infty} k^{2}\left(\frac{\left(a_{1}\right)_{k-1}\left(b_{1}\right)_{k-1}}{\left(c_{1}\right)_{k-1}(1)_{k-1}}+\frac{\left(a_{2}\right)_{k}\left(b_{2}\right)_{k}}{\left(c_{2}\right)_{k}(1)_{k}}\right) \\
& \leq 1-\beta,
\end{aligned}
$$

then $\frac{1}{A_{1}} G * F \in H P(\beta)$.

(iii) Let $G(z)=z+\overline{\frac{a_{2} b_{2}}{c_{2}}} z+\sum_{k=2}^{\infty}\left(\frac{\left(a_{1}\right)_{k-1}\left(b_{1}\right)_{k-1}}{\left(c_{1}\right)_{k-1}(1)_{k-1}} z^{k}+\overline{\frac{\left(a_{2}\right)_{k}\left(b_{2}\right)_{k}}{\left(c_{2}\right)_{k}(1)_{k}} z^{k}}\right)$ is a member

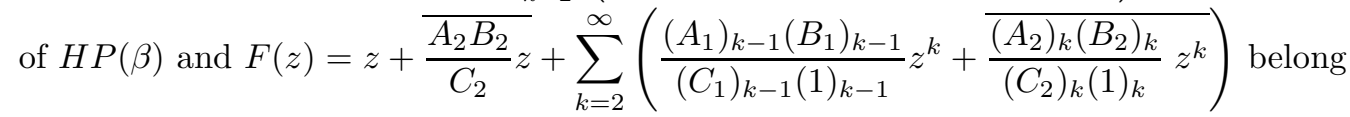
to $N_{\delta}(G)$. We have

$$
\begin{aligned}
\frac{A_{2} B_{2}}{C_{2}}+ & \sum_{k=2}^{\infty} k\left[\frac{\left(A_{1}\right)_{k-1}\left(B_{1}\right)_{k-1}}{\left(C_{1}\right)_{k-1}(1)_{k-1}}+\frac{\left(A_{2}\right)_{k}\left(B_{2}\right)_{k}}{\left(C_{2}\right)_{k}(1)_{k}}\right] \\
\leq & \left(\frac{A_{2} B_{2}}{C_{2}}-\frac{a_{2} b_{2}}{c_{2}}\right)+\frac{a_{2} b_{2}}{c_{2}}+\sum_{k=2}^{\infty} k\left[\left(\frac{\left(A_{1}\right)_{k-1}\left(B_{1}\right)_{k-1}}{\left(C_{1}\right)_{k-1}(1)_{k-1}}-\frac{\left(a_{1}\right)_{k-1}\left(b_{1}\right)_{k-1}}{\left(c_{1}\right)_{k-1}(1)_{k-1}}\right)\right. \\
& \left.+\left(\frac{\left(A_{2}\right)_{k}\left(B_{2}\right)_{k}}{\left(C_{2}\right)_{k}(1)_{k}}-\frac{\left(a_{2}\right)_{k}\left(b_{2}\right)_{k}}{\left(c_{2}\right)_{k}(1)_{k}}\right)\right] \\
& +\sum_{k=2}^{\infty} k\left(\frac{\left(a_{1}\right)_{k-1}\left(b_{1}\right)_{k-1}}{\left(c_{1}\right)_{k-1}(1)_{k-1}}+\frac{\left(a_{2}\right)_{k}\left(b_{2}\right)_{k}}{\left(c_{2}\right)_{k}(1)_{k}}\right)
\end{aligned}
$$




$$
\begin{aligned}
& \leq(1-\beta) \delta+\frac{a_{2} b_{2}}{c_{2}}+\frac{1}{2} \sum_{k=2}^{\infty} k^{2}\left(\frac{\left(a_{1}\right)_{k-1}\left(b_{1}\right)_{k-1}}{\left(c_{1}\right)_{k-1}(1)_{k-1}}+\frac{\left(a_{2}\right)_{k}\left(b_{2}\right)_{k}}{\left(c_{2}\right)_{k}(1)_{k}}\right) \\
& \leq(1-\beta) \delta+\frac{a_{2} b_{2}}{c_{2}}+\frac{1}{2}(1-\beta) \\
& \leq 1-\beta .
\end{aligned}
$$

Hence, for $\delta \leq \frac{1}{2}-\frac{1}{1-\beta} \frac{a_{2} b_{2}}{c_{2}}, F(z) \in H P(\beta)$.

\section{References}

[1] B. C. Carlson and D. B. Shaffer, Starlike and prestarlike hypergeometric functions, SIAM J. Math. Anal. 15 (1984), 737-745.

[2] E. Merkes and B. T. Scott, Starlike hypergeometric functions, Proc. American Math. Soc. 12 (1961), 885-888.

[3] H. M. Srivastava and H.L. Manocha, A treatise on generating functions, Ellis Harwood Limited and John Wiley and Sons, New York, Chichester, Brisbane, Toronto, 1984.

[4] J. Clunie and T. Sheil-Small, Harmonic univalent functions, Ann. Acad. Aci., Fenn. Ser A. I. Math. 9(1984), 3-25.

[5] O. Altintaş, Ö. özkan and H. M. Srivastava, Neighborhoods of a class of analaytic functions with negative coefficfients, Appl. Math. Lett. 13(2000), 63-67.

[6] O.P. Ahuja and H. Silverman, Inequalities associating hypergeometric functions with planer harmonic mappings, J. Inequal. Pure and Appl. Maths. 5 (2004), 1-10.

[7] St. Ruscheweyh, Neighborhoods of unilvalent functions, Proc. Amer. Math. Soc. 81(1981), 521-528.

[8] S. Yalçin, M. Öztürk and M. Yamankaradeniz, A subclass of harmonic univalent functions with negative coefficients, Appl. Math. Comput. 142(2003), 469-476.

[9] Z. J. Jakubowski, W. Majcharzak and K. Skalska, Harmonic mappings with a positive real part, Materialy XIV Konferencji z Teorii Zagadnien Ekstremalnych, Lodz. (1993), 17-24.

Department of Mathematics, Faculty of Science, Girls College, P.O. Box 838, Dammam, Saudi Arabia.

E-mail: hakh73@hotmail.com

Department of Mathematics, Faculty of Science, Girls College, P.O. Box 838, Dammam, Saudi Arabia.

E-mail: ranaab@hotmail.com 